\title{
Net soil-atmosphere fluxes mask patterns in gross production and consumption of nitrous oxide and methane in a managed ecosystem
}

\author{
Wendy H. Yang ${ }^{1, a}$ and Whendee L. Silver ${ }^{1}$ \\ ${ }^{1}$ Ecosystem Sciences Division, Department of Environmental Science, Policy, and Management, \\ 130 Mulford Hall \#3114, University of California, Berkeley, CA 94720, USA \\ ${ }^{a}$ now at: Departments of Plant Biology and Geology, 265 Morrill Hall, 505 South Goodwin Ave, \\ University of Illinois, Urbana, IL 61801, USA
}

Correspondence to: Wendy H. Yang (yangw@illinois.edu)

Received: 3 November 2015 - Published in Biogeosciences Discuss.: 4 December 2015

Revised: 1 March 2016 - Accepted: 9 March 2016 - Published: 18 March 2016

\begin{abstract}
Nitrous oxide $\left(\mathrm{N}_{2} \mathrm{O}\right)$ and methane $\left(\mathrm{CH}_{4}\right)$ are potent greenhouse gases that are both produced and consumed in soil. Production and consumption of these gases are driven by different processes, making it difficult to infer their controls when measuring only net fluxes. We used the trace gas pool dilution technique to simultaneously measure gross fluxes of $\mathrm{N}_{2} \mathrm{O}$ and $\mathrm{CH}_{4}$ throughout the growing season in a cornfield in northern California, USA. Net $\mathrm{N}_{2} \mathrm{O}$ fluxes ranged $0-4.5 \mathrm{mg} \mathrm{N} \mathrm{m}^{-2} \mathrm{~d}^{-1}$ with the $\mathrm{N}_{2} \mathrm{O}$ yield averaging $0.68 \pm 0.02$. Gross $\mathrm{N}_{2} \mathrm{O}$ production was best predicted by net nitrogen $(\mathrm{N})$ mineralization, soil moisture, and soil temperature $\left(R^{2}=0.60, n=39, p<0.001\right)$. Gross $\mathrm{N}_{2} \mathrm{O}$ reduction was correlated with the combination of gross $\mathrm{N}_{2} \mathrm{O}$ production rates, net $\mathrm{N}$ mineralization rates, and $\mathrm{CO}_{2}$ emissions $\left(R^{2}=0.74, n=39, p<0.001\right)$. Overall, net $\mathrm{CH}_{4}$ fluxes averaged $-0.03 \pm 0.02 \mathrm{mg} \mathrm{C} \mathrm{m}^{-2} \mathrm{~d}^{-1}$. The methanogenic fraction of carbon mineralization ranged from 0 to $0.27 \%$ and explained $40 \%$ of the variability in gross $\mathrm{CH}_{4}$ production rates $(n=37, p<0.001)$. Gross $\mathrm{CH}_{4}$ oxidation exhibited a strong positive relationship with gross $\mathrm{CH}_{4}$ production rates $\left(R^{2}=0.67, n=37, p<0.001\right)$, which reached as high as $5.4 \mathrm{mg} \mathrm{C} \mathrm{m}^{-2} \mathrm{~d}^{-1}$. Our study is the first to demonstrate the simultaneous in situ measurement of gross $\mathrm{N}_{2} \mathrm{O}$ and $\mathrm{CH}_{4}$ fluxes, and results highlight that net soil-atmosphere fluxes can mask significant gross production and consumption of these trace gases.
\end{abstract}

\section{Introduction}

Greenhouse gas emissions from soils are major contributors to climate change (Ciais et al., 2013). While carbon dioxide $\left(\mathrm{CO}_{2}\right)$ is the most abundant greenhouse gas in the atmosphere, both nitrous oxide $\left(\mathrm{N}_{2} \mathrm{O}\right)$ and methane $\left(\mathrm{CH}_{4}\right)$ are more potent with 298 and 34 times the global warming potential of $\mathrm{CO}_{2}$ on a 100-year timescale, respectively (Myhre et al., 2013). Both $\mathrm{N}_{2} \mathrm{O}$ and $\mathrm{CH}_{4}$ are produced and consumed in soils by microbially mediated redox-sensitive processes. However, most studies only measure net soil-atmosphere exchange of $\mathrm{N}_{2} \mathrm{O}$ and $\mathrm{CH}_{4}$. This approach cannot differentiate between production and consumption of these trace gases and thus limits our ability to infer controls on these processes and to diagnose model inaccuracies in predicting net $\mathrm{N}_{2} \mathrm{O}$ and $\mathrm{CH}_{4}$ fluxes. This hinders predictions of how soil-atmosphere $\mathrm{N}_{2} \mathrm{O}$ and $\mathrm{CH}_{4}$ fluxes will respond to future changes in land use practices or climate change.

Agricultural soils account for nearly two-thirds of global soil emissions of $\mathrm{N}_{2} \mathrm{O}$, which is produced from nitrification and denitrification of fertilizer nitrogen $(\mathrm{N})$ that supports agroecosystem productivity (Ciais et al., 2013). Managing soil $\mathrm{N}_{2} \mathrm{O}$ emissions from agroecosystems can go beyond direct reductions in $\mathrm{N}_{2} \mathrm{O}$ production from decreased fertilizer inputs because denitrifying bacteria can consume $\mathrm{N}_{2} \mathrm{O}$ to dinitrogen gas $\left(\mathrm{N}_{2}\right)$, completing the $\mathrm{N}$ cycling. Nitrous oxide consumption is not generally considered to be an important process in upland soils because it is an anaerobic process. Rates of $\mathrm{N}_{2} \mathrm{O}$ reduction to $\mathrm{N}_{2}$ decrease as $\mathrm{O}_{2}$ and $\mathrm{NO}_{3}^{-}$availability increases (Weier et al., 1993; Firestone et 
al., 1980). Theoretically, this results in a high $\mathrm{N}_{2} \mathrm{O}$ yield $\left(\mathrm{N}_{2} \mathrm{O} /\left(\mathrm{N}_{2} \mathrm{O}+\mathrm{N}_{2}\right)\right)$ in unsaturated soil where diffusive resupply of $\mathrm{O}_{2}$ and the production of $\mathrm{NO}_{3}^{-}$from nitrification would inhibit $\mathrm{N}_{2} \mathrm{O}$ reduction. Thermodynamics also predict that high soil $\mathrm{NO}_{3}^{-}$from fertilizer $\mathrm{N}$ inputs in agricultural soils would lead to high $\mathrm{N}_{2} \mathrm{O}$ yields. However, $\mathrm{N}_{2} \mathrm{O}$ yields average $0.375 \pm 0.035$ in agricultural soil and span the entire range from 0 to 1 in oxic, upland soils (Schlesinger, 2009; Stevens and Laughlin, 1998). This high variability, in part, reflects the difficulty in measuring rates of $\mathrm{N}_{2} \mathrm{O}$ reduction to $\mathrm{N}_{2}$, particularly under field conditions (Groffman et al., 2006). It also reflects other important factors that influence the $\mathrm{N}_{2} \mathrm{O}$ yield, such as soil type (Woli et al., 2010), labile C (Weier et al., 1993), and pH (Stevens et al., 1998). The lowerthan-expected average $\mathrm{N}_{2} \mathrm{O}$ yield in agricultural soils and large range in $\mathrm{N}_{2} \mathrm{O}$ yields in upland soil in general also suggests that $\mathrm{N}_{2} \mathrm{O}$ reduction to $\mathrm{N}_{2}$ could play an important role in mitigating soil $\mathrm{N}_{2} \mathrm{O}$ emissions to the atmosphere in agroecosystems.

Upland soils globally consume atmospheric $\mathrm{CH}_{4}$ at a rate similar to the accumulation of $\mathrm{CH}_{4}$ in the atmosphere (Ciais et al., 2013), and thus changes in the $\mathrm{CH}_{4}$ sink strength of soils could influence atmospheric $\mathrm{CH}_{4}$ concentrations. The inhibition of $\mathrm{CH}_{4}$ oxidation associated with fertilizer application of $\mathrm{NO}_{3}^{-}$(Aronson and Helliker, 2010), urea (Mosier et al., 1991), and $\mathrm{NH}_{4}^{+}$(Bedard and Knowles, 1989) is thought to cause lower net rates of $\mathrm{CH}_{4}$ uptake in agricultural systems compared to natural ecosystems (Nesbit and Breitenbeck, 1992; Bender and Conrad, 1994; Koschorreck and Conrad, 1993; Dutaur and Verchot, 2007; Mosier et al., 1991). Inhibition by $\mathrm{NH}_{4}^{+}$has been attributed to enzymatic substrate competition due to the similarities between the $\mathrm{CH}_{4}$ monooxygenase and $\mathrm{NH}_{4}^{+}$monooxygenase enzymes (Gulledge and Schimel, 1998) as well as toxicity effects from nitrite produced during $\mathrm{NH}_{4}^{+}$oxidation (King and Schnell, 1994). However, the effect of $\mathrm{N}$ on $\mathrm{CH}_{4}$ oxidation varies by soil (Gulledge et al., 1997), and at least some of this effect is due to inhibition by salts included in the fertilizer applications (Adamsen and King, 1993; Dunfield et al., 1993; Gulledge and Schimel, 1998; Nesbit and Breitenbeck, 1992). In addition, the response of $\mathrm{CH}_{4}$ oxidation to $\mathrm{NH}_{4}^{+}$and $\mathrm{NO}_{3}^{-}$may depend on the methanotrophic community; for example the high affinity type II methane-oxidizing bacteria that dominate under low $(<1000 \mathrm{ppm}) \mathrm{CH}_{4}$ conditions (Bender and Conrad, 1992) may be less sensitive to mineral $\mathrm{N}$ availability (Jang et al., 2011; Reay and Nedwell, 2004; Wang and Ineson, 2003). Thus, there remains uncertainty surrounding $\mathrm{N}$ inhibition of $\mathrm{CH}_{4}$ oxidation as the mechanism leading to low net rates of $\mathrm{CH}_{4}$ uptake in agricultural soils.

A major confounding factor in studies assessing controls on $\mathrm{CH}_{4}$ oxidation is the simultaneous occurrence of methanogenesis and $\mathrm{CH}_{4}$ oxidation. Net changes in $\mathrm{CH}_{4}$ concentrations under oxic soil conditions are assumed to reflect only $\mathrm{CH}_{4}$ oxidation (e.g., Nesbit and Breitenbeck,
1992) because methanogenesis occurs only under highly reducing conditions (Conrad, 1996). However, von Fischer and Hedin (2002) demonstrated that $\mathrm{CH}_{4}$ production occurred in a wide range of dry, oxic soils with water-filled pore space as low as $20 \%$. Similarly, Teh et al. (2005) documented the occurrence of methanogenesis under well-aerated conditions in an upland tropical forest soil. Macroaggregates can support net $\mathrm{CH}_{4}$ efflux in unsaturated soil (Jackel et al., 2001; Sey et al., 2008), likely because $\mathrm{O}_{2}$ consumption in the centers of the aggregates exceeds diffusive re-supply of $\mathrm{O}_{2}$ to create reducing conditions (Sexstone et al., 1985). Microsites of methanogenesis could also occur in the rhizosphere where high rates of $\mathrm{O}_{2}$ consumption from rhizosphere priming could create reducing conditions (Cheng et al., 2003). Because the controls on methanogenesis and $\mathrm{CH}_{4}$ oxidation are likely very different, the co-occurrence of these processes means that we must measure gross rates of both processes simultaneously to elucidate the mechanisms driving patterns in net soil-atmosphere $\mathrm{CH}_{4}$ fluxes.

We used the stable isotope trace gas pool dilution technique to measure gross $\mathrm{N}_{2} \mathrm{O}$ and $\mathrm{CH}_{4}$ fluxes in cornfield soils throughout the growing season in order to improve our understanding of trace gas dynamics in upland soils of agroecosystems. Fertilized agroecosystems are typically large net $\mathrm{N}_{2} \mathrm{O}$ sources and small net $\mathrm{CH}_{4}$ sinks (Haile-Mariam et al., 2008; Kessavalou et al., 1998; Gelfand et al., 2013; Nangia et al., 2013; Robertson et al., 2000). However, little is known about the rates of gross production and consumption of these gases in upland soils, or their controlling factors. Different controls on production and consumption processes may result in complex responses of net soil-atmosphere gas fluxes to climate or land management. Thus, the objectives of this study were to quantify field rates of gross $\mathrm{N}_{2} \mathrm{O}$ and $\mathrm{CH}_{4}$ production and consumption, and explore environmental and plant-mediated controls on these rates.

\section{Materials and methods}

\subsection{Study site}

The study site was a cornfield planted on a drained peatland located on Twitchell Island $\left(38.11^{\circ} \mathrm{N}, 121.65^{\circ} \mathrm{W}\right)$ in the Sacramento-San Joaquin River delta region of northern California. The region is very productive agriculturally, producing USD 500 million in crops in 1993 (Ingebritsen and Ikehara, 1999). The climate is Mediterranean with a winter wet season and summer dry season. The mean annual temperature is $15.1{ }^{\circ} \mathrm{C}$, and mean annual precipitation is $335 \mathrm{~mm}$ (Hatala et al., 2012). The soils consist of mucky clay over buried peat and are classified as fine, mixed, superactive, thermic Cumulic Endoaquolls (Drexler et al., 2009). The field was fertilized once, at seeding, at a rate of $118 \mathrm{~kg} \mathrm{Nha}^{-1}$ with UAN 32, which consists of $45 \%$ ammonium nitrate, $35 \%$ urea, and $20 \%$ water. The water table was 
maintained around $50 \mathrm{~cm}$ soil depth throughout the growing season via subsurface irrigation.

\subsection{Study design}

We measured gross and net fluxes of $\mathrm{CO}_{2}, \mathrm{CH}_{4}$, and $\mathrm{N}_{2} \mathrm{O}$ at five time points during the growing season from May to November 2012 on the following days after seeding (DAS): 11 (germination stage), 24 (seedling stage), 59 (peak growth stage), 94 (flowering stage), and 171 (senesced stage). The corn began senescing around DAS 104 and was harvested on DAS 178. We performed measurements in row and inter-row locations with the assumption that plant effects, if any, would be greater in the rows where the corn was growing (Cai et al., 2012; Haile-Mariam et al., 2008; Kessavalou et al., 1998). We established three parallel transects spaced $50 \mathrm{~m}$ apart. We measured gross production and consumption of $\mathrm{CH}_{4}$ and $\mathrm{N}_{2} \mathrm{O}$ as well as net fluxes of $\mathrm{CO}_{2}, \mathrm{CH}_{4}$, and $\mathrm{N}_{2} \mathrm{O}$ along the northernmost transect and measured only net fluxes in the other two transects. In each transect, we used paired measurements in the bed (in between corn rows) and furrow (in row) with replicate pairs spaced $10 \mathrm{~m}$ apart $(n=4$ pairs per transect). After each gas flux measurement was completed, we measured air, chamber headspace, and soil temperature at the surface flux chamber location. We also used an auger to sample the soil from the chamber footprint in $10 \mathrm{~cm}$ increments to $50 \mathrm{~cm}$ depth for the gross flux transect and only $0-10 \mathrm{~cm}$ depth in the net flux transects. The soils were processed the next day for determination of gravimetric soil moisture and net rates of nitrogen $(\mathrm{N})$ mineralization and nitrification as described below.

\subsection{Laboratory assays}

We determined net rates of $\mathrm{N}$ mineralization and nitrification from 6-day laboratory incubations. We mixed each soil core by hand and subsampled $15 \mathrm{~g}$ for extraction in $75 \mathrm{~mL}$ of $2 \mathrm{M}$ $\mathrm{KCl}, 10 \mathrm{~g}$ for determination of gravimetric soil moisture, and $50 \mathrm{~g}$ for incubation in Mason jars kept in the dark at ambient temperature. The jars were covered in perforated plastic wrap to minimize evaporation during the incubation. After 6 days, the soils in the jars were mixed and $15 \mathrm{~g}$ of soil was subsampled for $\mathrm{KCl}$ extractions. The $\mathrm{KCl}$ extracts were analyzed colorimetrically for $\mathrm{NH}_{4}^{+}$and $\mathrm{NO}_{3}^{-}$concentrations on a Lachat QuickChem flow injection auto-analyzer (Lachat Instruments, Milwaukee, WI, USA). We calculated net N mineralization rates from the change in $\mathrm{NH}_{4}^{+}$plus $\mathrm{NO}_{3}^{-}$concentrations over the incubation period and net nitrification rates from the change in $\mathrm{NO}_{3}^{-}$concentrations over the incubation period.

The remaining soil (not utilized in the net rates incubation) was air dried for archival. Air-dried samples from the May sampling date were ground in a Spex Mill (Metuchen, NJ, USA) for total $\mathrm{C}$ and $\mathrm{N}$ analyses on a vario MICRO cube elemental analyzer (Elementar, Hanau, Germany).

\subsection{Gas flux measurements}

We used the stable isotope trace gas pool dilution technique to measure field rates of gross $\mathrm{N}_{2} \mathrm{O}$ and $\mathrm{CH}_{4}$ production and consumption (von Fischer and Hedin, 2002; Yang et al., 2011). We injected $10 \mathrm{~mL}$ of isotopically enriched spiking gas into the headspace of a $28 \mathrm{~L}$ surface flux chamber inserted $6 \mathrm{~cm}$ into the soil surface. The spiking gas consisted of $70 \mathrm{ppm} \mathrm{N}_{2} \mathrm{O}$ at 98 atom $\%{ }^{15} \mathrm{~N}$ enrichment, $280 \mathrm{ppm} \mathrm{CH}_{4}$ at 99 atom $\%{ }^{13} \mathrm{C}$ enrichment, and $28 \mathrm{ppm} \mathrm{\textrm {SF } _ { 6 }}$ to achieve a ${ }^{15} \mathrm{~N}-\mathrm{N}_{2} \mathrm{O}$ enrichment of 5.42 atom $\%$ and ${ }^{13} \mathrm{C}_{-} \mathrm{CH}_{4}$ enrichment of 5.61 atom \%. This spiking gas injection increased the chamber headspace gas composition by $25 \mathrm{~N}_{2} \mathrm{O}, 100$ $\mathrm{CH}_{4}$, and $10 \mathrm{ppb} \mathrm{SF}_{6}$. We sampled the chamber headspace at 5, 15, 30, 45, and $60 \mathrm{~min}$ after spiking gas injection. We analyzed samples on a Shimadzu GC-14A gas chromatograph (Columbia, MD, USA) equipped with a thermal conductivity detector, flame ionization detector, and electron capture detector for determination of $\mathrm{CO}_{2}, \mathrm{CH}_{4}, \mathrm{~N}_{2} \mathrm{O}$, and $\mathrm{SF}_{6}$ concentrations. We analyzed separate samples for ${ }^{15} \mathrm{~N}$ $\mathrm{N}_{2} \mathrm{O}$ and ${ }^{13} \mathrm{C}_{-} \mathrm{CH}_{4}$ on an IsoPrime 100 continuous flow isotope ratio mass spectrometer interfaced with a trace gas preconcentration unit (Isoprime Ltd, Cheadle Hulme, UK) and Gilson GX271 autosampler (Middleton, WI). The trace gas analyzer was equipped with a combustion furnace using palladium to catalyze the conversion of $\mathrm{CH}_{4}$ to $\mathrm{CO}_{2}$ for isotopic analysis after $\mathrm{CO}$ and $\mathrm{CO}_{2}$ were scrubbed from the sample (Fisher et al., 2006). One out of the 40 gross $\mathrm{N}_{2} \mathrm{O}$ flux measurements and three out of the 40 gross $\mathrm{CH}_{4}$ flux measurements were lost due to autosampler needle clogs that occurred during isotopic analysis.

Gross $\mathrm{N}_{2} \mathrm{O}$ and $\mathrm{CH}_{4}$ production and consumption rates were estimated using the pool dilution model as described by Yang et al. (2011) and von Fischer and Hedin (2002). The iterative model solves for gross production rates based on the isotopic dilution of the isotopically enriched chamber headspace pool of $\mathrm{N}_{2} \mathrm{O}$ or $\mathrm{CH}_{4}$ by natural abundance $\mathrm{N}_{2} \mathrm{O}$ or $\mathrm{CH}_{4}$ emitted by the soil. Gross consumption rates were estimated from the empirical loss of the ${ }^{15} \mathrm{~N}_{2} \mathrm{O}$ or ${ }^{13} \mathrm{CH}_{4}$ tracer, using the loss of the $\mathrm{SF}_{6}$ tracer to account for physical losses such as diffusion. We note that gross $\mathrm{N}_{2} \mathrm{O}$ consumption rates are not equivalent to $\mathrm{N}_{2}$ production via denitrification because heterogeneous ${ }^{15} \mathrm{~N}_{2} \mathrm{O}$ distribution in the soil and complete denitrification intracellularly could lead to underestimates of $\mathrm{N}_{2}$ production (Well and Butterbach-Bahl, 2013; Yang et al., 2013). We assumed that the isotopic composition of produced $\mathrm{N}_{2} \mathrm{O}$ was 0.3431 atom $\%{ }^{15} \mathrm{~N}$ and the fractionation factor associated with $\mathrm{N}_{2} \mathrm{O}$ reduction to $\mathrm{N}_{2}$ was 0.9924 . The justification for these assumptions is discussed by Yang et al. (2011). We assumed that the isotopic composition of produced $\mathrm{CH}_{4}$ was 1.0473 atom \%, based on measurements of the ${ }^{13} \mathrm{C}$ isotopic composition of soil $\mathrm{CH}_{4}$ in a nearby study site (Y. Teh, personal communication, 2011). We assumed that the fractionation factor associated with $\mathrm{CH}_{4}$ oxidation was 0.98 as justified by von Fischer and Hedin (2002). Sen- 
sitivity analyses performed by both Yang et al. (2011) and von Fischer and Hedin (2002) showed that the pool dilution model output is not sensitive to these assumed values at the high isotopic enrichments used. Net fluxes of $\mathrm{CO}_{2}, \mathrm{~N}_{2} \mathrm{O}$, and $\mathrm{CH}_{4}$ were determined from the change in concentration over time using an iterative model that fits an exponential curve to the data (Matthias et al., 1978). Fluxes were considered to be zero when the relationship between trace gas concentration and time was not significant at $p=0.05$. The methanogenic fraction of $\mathrm{C}$ mineralization was calculated as the gross $\mathrm{CH}_{4}$ production rate divided by the sum of the gross $\mathrm{CH}_{4}$ production rate and $\mathrm{CO}_{2}$ production rates.

\subsection{Statistical analyses}

We used SYSTAT version 13 (SPSS Inc., Evanston, IL, USA) to perform statistical analyses and Microsoft Excel 2007 (Microsoft Corporation, Redmond, WA, USA) to run the iterative pool dilution model. We log transformed the data to meet the normality assumptions of ANOVAs; soil moisture, soil temperature, soil $\mathrm{C}$ and $\mathrm{N}$ concentrations, and soil $\mathrm{C}: \mathrm{N}$ ratios did not require transformation. We analyzed net and gross fluxes of $\mathrm{CO}_{2}, \mathrm{~N}_{2} \mathrm{O}$, and $\mathrm{CH}_{4}$ using sampling date as the within-subjects factor and location (i.e., bed versus furrow) as the between-subject factor in repeated measures ANOVAs. We also analyzed net $\mathrm{N}$ mineralization and nitrification rates using sampling date as the within-subjects factor, and soil depth and location as the between-subjects factors in repeated measures ANOVAs. We explored relationships between trace gas fluxes and potential drivers (soil moisture, air and soil temperatures, soil $\mathrm{NH}_{4}^{+}$and $\mathrm{NO}_{3}^{-}$concentrations, net $\mathrm{N}$ mineralization and nitrification rates, soil $\mathrm{C}$ and $\mathrm{N}$ concentrations, etc.) using linear regressions. We determined the model that best fit observed trace gas flux data using backwards stepwise multiple linear regressions starting with all potential explanatory variables; the best model fit was determined by minimizing the Akaike information criterion. Statistical significance was determined at $p$ values $<0.05$.

\section{Results}

\subsection{Soil characteristics and $N$ cycling}

Air and soil temperature differed significantly among sampling dates $(p<0.05$, Table 1$)$. Mean air temperature spanned a small range from a low of $24.5 \pm 0.7^{\circ} \mathrm{C}$ on DAS 171 to a high of $28.2 \pm 0.7^{\circ} \mathrm{C}$ on DAS 94. Soil temperature was more variable, with the lowest mean soil temperature on DAS 171 at $14.8 \pm 0.1^{\circ} \mathrm{C}$ and the highest mean soil temperature on DAS 59 at $24.2 \pm 0.3^{\circ} \mathrm{C}$.

In surface soils $(0-10 \mathrm{~cm}$ depth), gravimetric soil moisture ranged from $0.24 \pm 0.01 \mathrm{~g} \mathrm{H}_{2} \mathrm{O} \mathrm{g}^{-1}$ soil on DAS 94 to $0.38 \pm 0.02 \mathrm{~g} \mathrm{H}_{2} \mathrm{O} \mathrm{g}^{-1}$ soil on DAS 11 (Table 1). Soil moisture decreased as the growing season progressed until DAS 171, when soil moisture increased to a value intermediate of that on DAS 59 and 94 (Table 1). Soil moisture was significantly higher in the row than in the inter-row on DAS 11 and 24 only (Table 3). Mean soil moisture increased significantly with depth (Table 2), although differences were not statistically significant for all dates (Table 3).

Soil $\mathrm{NH}_{4}^{+}$concentrations differed significantly among sampling dates and were lowest at the beginning (DAS 11) and end (DAS 171) of the study (Table 3). The inclusion of an outlier plot on DAS 94 increased the mean $\mathrm{NH}_{4}^{+}$concentration to $62.5 \pm 46.4 \mu \mathrm{g} \mathrm{N} \mathrm{g}-1$. Soil $\mathrm{NH}_{4}^{+}$concentrations decreased significantly from $0-10$ to $20-30 \mathrm{~cm}$ depth (Tables 2-3) and were higher in rows than inter-rows (Table 3). Across all sampling dates, concentrations at $0-10 \mathrm{~cm}$ depth averaged $34.8 \pm 20.1$ in rows and $12.9 \pm 2.0 \mu \mathrm{g} \mathrm{N} \mathrm{g}-1$ in inter-rows. Soil $\mathrm{NO}_{3}^{-}$concentrations were lower on DAS 11 than all other sampling dates (Table 3), averaging $53.5 \pm 7.2$ on DAS 11 and $215 \pm 33 \mu \mathrm{g} \mathrm{N} \mathrm{g}^{-1}$ across all other sampling dates at $0-10 \mathrm{~cm}$ depth. Soil $\mathrm{NO}_{3}^{-}$concentrations decreased with depth (Tables 2-3). On DAS 59 and 94 only, soil $\mathrm{NO}_{3}$ concentrations were higher in rows $(387 \pm 117 \mu \mathrm{g} \mathrm{N} \mathrm{g}-1)$ than in inter-rows $\left(156 \pm 23 \mu \mathrm{g} \mathrm{N} \mathrm{g}^{-1}\right)$ (Table 3).

Across the entire data set $(n=216)$, net $\mathrm{N}$ mineralization rates averaged $3.3 \pm 0.5$ and net nitrification rates averaged $2.7 \pm 0.6 \mu \mathrm{g} \mathrm{N} \mathrm{g}^{-1} \mathrm{~d}^{-1}$. Net $\mathrm{N}$ mineralization and nitrification rates did not differ significantly among soil depths, sampling locations, or sampling dates (Table 3), although rates trended higher at $0-10 \mathrm{~cm}$ depth across all sampling dates and locations (Table 2). Across all sampling dates and soil depths, $96 \%$ of the variability in net nitrification rates was explained by net $\mathrm{N}$ mineralization rates $(p<0.001, n=215$, Table 4).

Total $\mathrm{C}$ and $\mathrm{N}$ concentrations for soils sampled on DAS 11 differed between row and inter-row sampling locations (soil C, $F_{1,30}=5.295, p=0.03$; soil $\mathrm{N}, F_{1,30}=4.546$, $p=0.04$ ) but not among soil depths (Table 2). Both soil C and $\mathrm{N}$ concentrations were higher in rows than in inter-rows, averaging $16.1 \pm 0.8 \% \mathrm{C}$ and $0.99 \pm 0.03 \% \mathrm{~N}$ in rows and $13.7 \pm 0.5 \% \mathrm{C}$ and $0.89 \pm 0.02 \% \mathrm{~N}$ in inter-rows. Soil $\mathrm{C}: \mathrm{N}$ ratios averaged $15.8 \pm 0.2$ overall $(n=40)$, and did not differ significantly between sampling locations or among soil depths.

\subsection{Gross and net $\mathrm{N}_{2} \mathrm{O}$ fluxes}

Across the entire data set, net $\mathrm{N}_{2} \mathrm{O}$ fluxes ranged $0-4.5$ and averaged $1.6 \pm 0.2 \mathrm{mg} \mathrm{N} \mathrm{m}^{-2} \mathrm{~d}^{-1}(n=112)$. Net $\mathrm{N}_{2} \mathrm{O}$ fluxes differed significantly among sampling dates $\left(F_{4,56}=3.0\right.$, $p=0.03$ ) but not between sampling locations (Fig. 1a). Net $\mathrm{N}_{2} \mathrm{O}$ fluxes were best predicted by net $\mathrm{N}$ mineralization, soil moisture, and soil $\mathrm{CO}_{2}$ emissions together $\left(R^{2}=0.49\right.$, Table 4).

Gross $\mathrm{N}_{2} \mathrm{O}$ production ranged $0.09-6.6 \mathrm{mg} \mathrm{N} \mathrm{m}^{-2} \mathrm{~d}^{-1}$ and gross $\mathrm{N}_{2} \mathrm{O}$ reduction rates ranged $0.00-0.95 \mathrm{mg} \mathrm{N} \mathrm{m}^{-2} \mathrm{~d}^{-1}$. The $\mathrm{N}_{2} \mathrm{O}$ yield averaged $0.68 \pm 0.02 \quad(n=40)$. Both gross $\mathrm{N}_{2} \mathrm{O}$ production and consumption rates differed sig- 
Table 1. Environmental and soil $(0-10 \mathrm{~cm}$ depth) variables by sampling date (mean $\pm \mathrm{SE})$.

\begin{tabular}{lllllll}
\hline Variable & $F$ statistic & \multicolumn{5}{c}{ Sampling date } \\
\hline & & $\begin{array}{l}\text { DAS 11 } \\
(n=8)\end{array}$ & $\begin{array}{l}\text { DAS 24 } \\
(n=24)\end{array}$ & $\begin{array}{l}\text { DAS 59 } \\
(n=24)\end{array}$ & $\begin{array}{l}\text { DAS 94 } \\
(n=16)\end{array}$ & $\begin{array}{l}\text { DAS 171 } \\
(n=24)\end{array}$ \\
\hline Air temperature $\left({ }^{\circ} \mathrm{C}\right)$ & $F_{4,56}=\mathbf{3 . 4}$ & $27.6 \pm 0.9 \mathrm{a}$ & $26.8 \pm 1.2 \mathrm{a}$ & $25.8 \pm 0.5 \mathrm{ab}$ & $28.2 \pm 0.7 \mathrm{a}$ & $24.5 \pm 0.7 \mathrm{~b}$ \\
Soil temperature $\left({ }^{\circ} \mathrm{C}\right)$ & $F_{4,56}=\mathbf{2 4 5}$ & $18.4 \pm 0.2 \mathrm{a}$ & $22.1 \pm 0.3 \mathrm{~b}$ & $24.2 \pm 0.3 \mathrm{c}$ & $22.8 \pm 0.2 \mathrm{~b}$ & $14.8 \pm 0.1 \mathrm{~d}$ \\
Soil moisture $\left(\mathrm{g} \mathrm{H}_{2} \mathrm{O} \mathrm{g}^{-1}\right.$ soil $)$ & $F_{4,24}=\mathbf{3 4}^{*}$ & $0.38 \pm 0.02$ & $0.34 \pm 0.02$ & $0.35 \pm 0.01$ & $0.24 \pm 0.01$ & $0.32 \pm 0.01$ \\
\hline
\end{tabular}

Degrees of freedom are shown in subscripts, and statistically significant $F$ statistics at $P<0.05$ are indicated by bold text. Letters indicate statistically significant differences among sampling dates. ${ }^{*}$ One transect was excluded from the repeated measures ANOVA because data are missing for one sampling date.

Table 2. Soil characteristics and $\mathrm{N}$ cycling rates across all sampling dates by soil depth in the gross flux transect (mean \pm SE).

\begin{tabular}{llllllll}
\hline Variable & $\mathrm{N}$ & $P$ value & $0-10 \mathrm{~cm}$ & $10-20 \mathrm{~cm}$ & $20-30 \mathrm{~cm}$ & $30-40 \mathrm{~cm}$ & $40-50 \mathrm{~cm}$ \\
\hline Soil moisture $\left(\mathrm{g} \mathrm{H}_{2} \mathrm{O} \mathrm{g}^{-1}\right)$ & 40 & $<0.001$ & $0.34 \pm 0.01 \mathrm{a}$ & $0.35 \pm 0.01 \mathrm{a}$ & $0.37 \pm 0.01 \mathrm{ab}$ & $0.40 \pm 0.01 \mathrm{~b}$ & $0.46 \pm 0.02 \mathrm{c}$ \\
$\mathrm{NH}_{4}^{+}$concentration $\left(\mu \mathrm{g} \mathrm{N} \mathrm{g}^{-1}\right)$ & 40 & $<0.001$ & $23.3 \pm 9.6 \mathrm{a}$ & $15.2 \pm 6.3 \mathrm{~b}$ & $7.0 \pm 1.8 \mathrm{~b}$ & $5.0 \pm 0.7 \mathrm{~b}$ & $5.7 \pm 0.8 \mathrm{~b}$ \\
$\mathrm{NO}_{3}^{-}$concentration $\left(\mu \mathrm{g} \mathrm{N} \mathrm{g}^{-1}\right)$ & 40 & $<0.001$ & $183 \pm 28 \mathrm{a}$ & $110 \pm 22 \mathrm{~b}$ & $58.9 \pm 8.4 \mathrm{c}$ & $41.9 \pm 6.1 \mathrm{c}$ & $29.5 \pm 3.2 \mathrm{c}$ \\
$\mathrm{Net}_{\text {mineralization }}\left(\mu \mathrm{g} \mathrm{N} \mathrm{g}^{-1} \mathrm{~d}^{-1}\right)$ & 40 & & $5.9 \pm 2.6$ & $1.0 \pm 0.9$ & $1.5 \pm 0.5$ & $1.8 \pm 0.7$ & $3.3 \pm 0.5$ \\
Net nitrification $\left(\mu \mathrm{g} \mathrm{g}^{-1} \mathrm{~d}^{-1}\right)$ & 40 & & $6.7 \pm 2.4$ & $1.7 \pm 0.8$ & $2.1 \pm 0.5$ & $2.3 \pm 0.7$ & $3.8 \pm 0.5$ \\
Soil C concentration $(\%)$ & $8^{*}$ & & $14.1 \pm 0.5$ & $15.4 \pm 1.6$ & $14.8 \pm 0.9$ & $15.2 \pm 1.6$ & $15.0 \pm 0.9$ \\
Soil N concentration $(\%)$ & $8^{*}$ & & $0.93 \pm 0.02$ & $0.96 \pm 0.07$ & $0.98 \pm 0.05$ & $0.91 \pm 0.06$ & $0.93 \pm 0.05$ \\
\hline
\end{tabular}

Letters indicate statistically significant differences among soil depths. * Data from DAS 11 only.
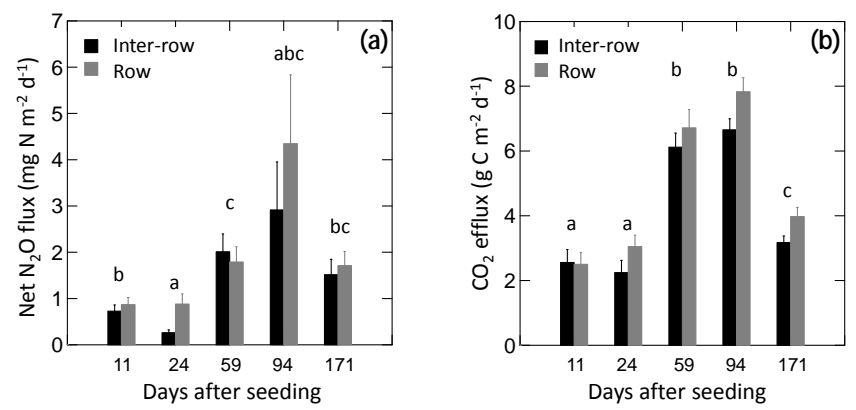

Figure 1. Mean (a) net $\mathrm{N}_{2} \mathrm{O}$ flux and (b) $\mathrm{CO}_{2}$ efflux for all three transects ( $n=24$ per sampling date except $n=16$ on DAS 94) in inter-rows (black bars) and rows (grey bars). Error bars represent standard errors, and different letters indicate statistically significant differences among sampling dates.

nificantly among sampling dates $\left(F_{4,20}=4.5, p=0.009\right.$ and $F_{4,20}=4.4, p=0.01$, respectively) but not between sampling locations (Fig. 2a). The highest gross production and consumption rates occurred on DAS 59 and 171. Overall, gross $\mathrm{N}_{2} \mathrm{O}$ production rates were best predicted by net $\mathrm{N}$ mineralization, soil moisture, soil temperature, and soil $\mathrm{CO}_{2}$ emissions $\left(R^{2}=0.60\right.$, Table 4$)$. At peak growth (DAS 59 and 94), these variables explained $89 \%$ of the variability in gross $\mathrm{N}_{2} \mathrm{O}$ production rates $(n=15, p<0.001)$. When the corn was not actively growing, gross $\mathrm{N}_{2} \mathrm{O}$ production was most strongly correlated with $\mathrm{CO}_{2}$ emissions alone $\left(R^{2}=0.68, n=24, p<0.001\right)$.
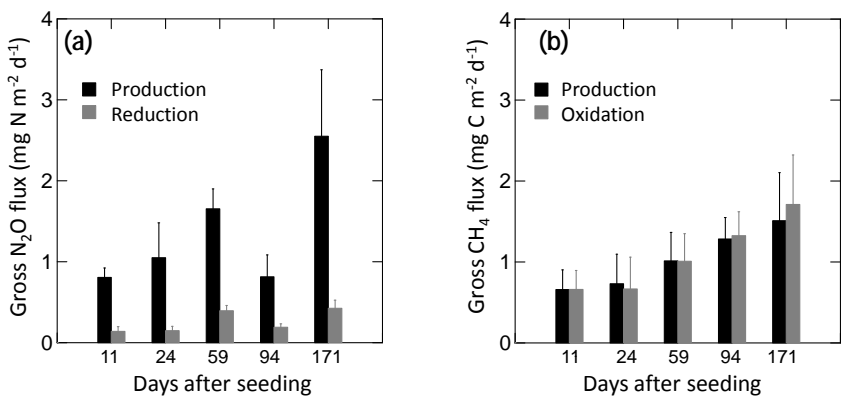

Figure 2. Mean (a) gross $\mathrm{N}_{2} \mathrm{O}$ production rates (black bars) and reduction rates (grey bars) and (b) gross $\mathrm{CH}_{4}$ production rates (black bars) and oxidation rates (grey bars). Error bars represent standard errors ( $n=8$ per sampling date).

Gross $\mathrm{N}_{2} \mathrm{O}$ reduction rates increased with gross $\mathrm{N}_{2} \mathrm{O}$ production rates $\left(R^{2}=0.60, n=39, p<0.001\right.$, Fig. 3a). Rates were also positively correlated with soil $\mathrm{CO}_{2}$ emissions $\left(R^{2}=0.36, n=39, p<0.001\right)$; this relationship was stronger when the corn was not actively growing (DAS 11, 24 , and 171), with $80 \%$ of the variability in gross $\mathrm{N}_{2} \mathrm{O}$ reduction rates explained by $\mathrm{CO}_{2}$ emissions on these dates $\left(n=24, p<0.001\right.$, Fig. 3b). Gross $\mathrm{N}_{2} \mathrm{O}$ reduction was most strongly correlated with the combination of gross $\mathrm{N}_{2} \mathrm{O}$ production rates, net $\mathrm{N}$ mineralization rates, and $\mathrm{CO}_{2}$ emissions $\left(R^{2}=0.74, n=39, p<0.001\right.$, Table 4). 
Table 3. Results from repeated measures ANOVAs with sampling date, the interaction of sampling date and soil depth, and the interaction of sampling date and sampling location as the within-subjects, and soil depth and sampling location as the between-subjects factors.

\begin{tabular}{|c|c|c|c|c|c|}
\hline & Sampling date & Soil depth & $\begin{array}{l}\text { Sampling } \\
\text { location }\end{array}$ & $\begin{array}{l}\text { Sampling date* } \\
\text { Soil depth }\end{array}$ & $\begin{array}{l}\text { Sampling date } \\
\text { Sampling } \\
\text { location }\end{array}$ \\
\hline Soil moisture $\left(\mathrm{g} \mathrm{H}_{2} \mathrm{Og}^{-1}\right)$ & $F_{4,120}=\mathbf{1 3 5}$ & $F_{4,30}=\mathbf{3 1}$ & $F_{1,30}=\mathbf{5 . 1}$ & $F_{16,120}=\mathbf{1 . 9}$ & $F_{4,120}=\mathbf{4 . 6}$ \\
\hline $\mathrm{NH}_{4}^{+}$concentration $\left(\mu \mathrm{g} \mathrm{Ng}^{-1} \mathrm{~d}^{-1}\right)$ & $F_{4,120}=\mathbf{7 . 9}$ & $F_{4,30}=7.7$ & $F_{1,30}=\mathbf{4 . 0}$ & $F_{16,120}=0.90$ & $F_{4,120}=1.9$ \\
\hline $\mathrm{NO}_{3}^{-}$concentration $\left(\mu \mathrm{g} \mathrm{Ng}^{-1} \mathrm{~d}^{-1}\right)$ & $F_{4,120}=\mathbf{1 7}$ & $F_{4,30}=\mathbf{3 6}$ & $F_{1,30}=\mathbf{1 8}$ & $F_{16,120}=1.0$ & $F_{4,120}=\mathbf{7 . 1}$ \\
\hline Net mineralization $\left(\mu \mathrm{g} \mathrm{Ng}-1 \mathrm{~d}^{-1}\right)$ & $F_{4,120}=1.5$ & $F_{4,30}=1.5$ & $F_{1,30}=1.5$ & $F_{16,120}=1.1$ & $F_{4,120}=1.7$ \\
\hline Net nitrification $\left(\mu \mathrm{g} \mathrm{N}^{-1} \mathrm{~d}^{-1}\right)$ & $F_{4,120}=1.4$ & $F_{4,30}=0.22$ & $F_{1,30}=1.9$ & $F_{16,120}=0.31$ & $F_{4,120}=1.8$ \\
\hline
\end{tabular}

Degrees of freedom are shown in subscripts, and statistically significant $F$ statistics at $P<0.05$ are indicated by bold text.

Table 4. Coefficients for multiple linear regressions predicting trace gas fluxes using soil variables.

\begin{tabular}{|c|c|c|c|c|c|c|}
\hline Dependent variable & $\mathrm{N}$ & $R^{2}$ & Effect & Coefficient & SE & $P$ value \\
\hline \multirow[t]{2}{*}{$\log \left(\right.$ Net nitrification, $\left.\mu \mathrm{g} \mathrm{Ng}^{-1} \mathrm{~d}^{-1}\right)$} & \multirow[t]{2}{*}{215} & \multirow[t]{2}{*}{0.96} & Constant & 0.162 & 0.020 & $<0.001$ \\
\hline & & & $\log \left(\right.$ Net $\mathrm{N}$ mineralization, $\left.\mu \mathrm{g} \mathrm{Ng}^{-1} \mathrm{~d}^{-1}\right)$ & 0.906 & 0.012 & $<0.001$ \\
\hline \multirow[t]{3}{*}{$\log \left(\mathrm{CO}_{2}\right.$ emissions, $\left.\mathrm{g} \mathrm{C}^{-2} \mathrm{~d}^{-1}\right)$} & \multirow[t]{3}{*}{96} & \multirow[t]{3}{*}{0.15} & Constant & 0.466 & 0.195 & 0.02 \\
\hline & & & Soil moisture $\left(\mathrm{g} \mathrm{H}_{2} \mathrm{Og}^{-1}\right)$ & -1.019 & 0.416 & 0.02 \\
\hline & & & Soil temperature $\left({ }^{\circ} \mathrm{C}\right)$ & 0.022 & 0.007 & 0.002 \\
\hline \multirow[t]{4}{*}{$\log \left(\right.$ Net $\mathrm{N}_{2} \mathrm{O}$ flux, $\left.\mathrm{mg} \mathrm{N} \mathrm{m}^{-2} \mathrm{~d}^{-1}\right)$} & \multirow[t]{4}{*}{56} & \multirow[t]{4}{*}{0.49} & Constant & -1.671 & 0.743 & 0.03 \\
\hline & & & $\log ($ Net $N$ mineralization $)$ & 1.342 & 0.274 & $<0.001$ \\
\hline & & & Soil moisture & 4.356 & 1.539 & 0.007 \\
\hline & & & $\log \left(\mathrm{CO}_{2}\right.$ emissions $)$ & 1.404 & 0.288 & $<0.001$ \\
\hline \multirow{5}{*}{$\log \left(\right.$ Gross $\mathrm{N}_{2} \mathrm{O}$ production, $\left.\mathrm{mg} \mathrm{N} \mathrm{m}^{-2} \mathrm{~d}^{-1}\right)$} & \multirow[t]{5}{*}{39} & \multirow[t]{5}{*}{0.60} & Constant & 1.743 & 0.453 & 0.001 \\
\hline & & & Log(Net N mineralization) & 0.516 & 0.139 & 0.001 \\
\hline & & & Soil moisture & 2.226 & 0.839 & 0.01 \\
\hline & & & Soil temperature & -0.043 & 0.013 & 0.002 \\
\hline & & & $\log \left(\mathrm{CO}_{2}\right.$ emissions $)$ & 1.056 & 0.180 & $<0.001$ \\
\hline \multirow{4}{*}{$\log \left(\right.$ Gross $\mathrm{N}_{2} \mathrm{O}$ reduction $)$} & \multirow[t]{4}{*}{39} & \multirow[t]{4}{*}{0.74} & Constant & -2.525 & 0.556 & $<0.001$ \\
\hline & & & $\log ($ Net $N$ mineralization $)$ & 0.680 & 0.258 & 0.01 \\
\hline & & & $\log \left(\right.$ Gross $\mathrm{N}_{2} \mathrm{O}$ production $)$ & 0.983 & 0.226 & $<0.001$ \\
\hline & & & $\log \left(\mathrm{CO}_{2}\right.$ emissions $)$ & 1.199 & 0.292 & $<0.001$ \\
\hline \multirow[t]{2}{*}{$\log \left(\right.$ Gross $\mathrm{CH}_{4}$ production, $\left.\mathrm{mg} \mathrm{C} \mathrm{m}^{-2} \mathrm{~d}^{-1}\right)$} & \multirow[t]{2}{*}{37} & \multirow[t]{2}{*}{0.17} & Constant & 2.264 & 0.199 & $<0.001$ \\
\hline & & & $\log \left(\mathrm{CO}_{2}\right.$ emissions $)$ & 0.921 & 0.348 & 0.01 \\
\hline \multirow[t]{4}{*}{$\log \left(\right.$ Gross $\mathrm{CH}_{4}$ oxidation $)$} & \multirow[t]{4}{*}{37} & \multirow[t]{4}{*}{0.79} & Constant & 0.794 & 0.621 & 0.21 \\
\hline & & & $\log \left(\right.$ Gross $\mathrm{CH}_{4}$ production $)$ & 1.090 & 0.142 & 0.002 \\
\hline & & & Soil temperature & -0.086 & 0.024 & 0.001 \\
\hline & & & $\log \left(\mathrm{CO}_{2}\right.$ emissions $)$ & 1.096 & 0.335 & $<0.001$ \\
\hline
\end{tabular}

\subsection{Gross and net $\mathrm{CH}_{4}$ fluxes}

Net $\mathrm{CH}_{4}$ fluxes ranged from -1.3 to $0.44 \mathrm{mg} \mathrm{C} \mathrm{m}^{-2} \mathrm{~d}^{-1}$ but net fluxes were not detectable for 94 out of 112 measurements. Overall net $\mathrm{CH}_{4}$ fluxes averaged $-0.03 \pm 0.02 \mathrm{mg} \mathrm{C} \mathrm{m}^{-2} \mathrm{~d}^{-1}$. Using the trace gas pool dilution technique, we detected gross $\mathrm{CH}_{4}$ production in 36 out of 37 measurements. Gross $\mathrm{CH}_{4}$ production reached as high as $5.4 \mathrm{mg} \mathrm{C} \mathrm{m}^{-2} \mathrm{~d}^{-1}$ with rates trending higher throughout the growing season (Fig. 2b). However, rates were only significantly different between DAS 11 and 94
$\left(F_{4,12}=4.1, p=0.03\right)$. Gross $\mathrm{CH}_{4}$ production rates were marginally significantly higher in rows than in inter-rows $\left(F_{1,3}=5.8, p=0.10\right)$. Overall, gross $\mathrm{CH}_{4}$ production rates were weakly correlated to soil $\mathrm{CO}_{2}$ emissions $\left(R^{2}=0.17\right.$, Table 4) but exhibited a stronger positive correlation with the methanogenic fraction of $\mathrm{C}$ mineralization $\left(R^{2}=0.40\right.$, $n=37, p<0.001$, Fig. 4 a), which ranged from 0 to $0.27 \%$ and averaged $0.06 \pm 0.01 \%$. The strength of the relationship increased to $R^{2}=0.60(n=23, p<0.001)$ when considering only dates when the corn was not actively growing (Fig. 4a). When only peak growth sampling dates were con- 

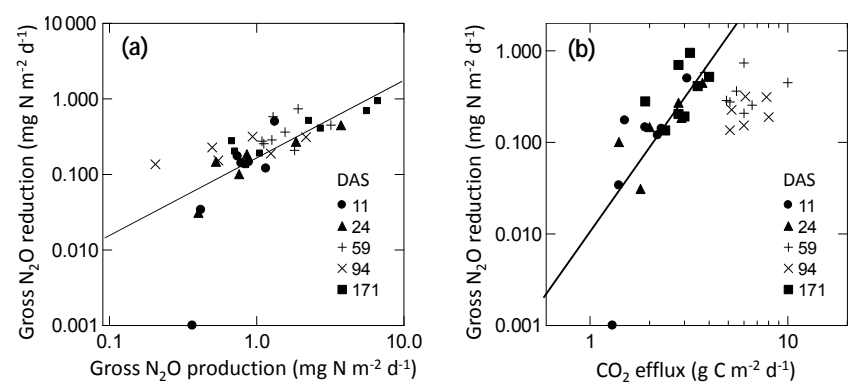

Figure 3. Gross $\mathrm{N}_{2} \mathrm{O}$ reduction rates versus (a) gross $\mathrm{N}_{2} \mathrm{O}$ production and (b) $\mathrm{CO}_{2}$ efflux. Symbols represent sampling on different days after seeding (DAS): circles are DAS 11, triangles are DAS 24, pluses are DAS 59, crosses are DAS 94, and squares are DAS 171. (a) The line represents the regression line for all sampling dates together, $\log _{10}(y)=\left[1.567 \times \log _{10}(x)\right]-2.573\left(R^{2}=0.60\right.$, $n=39, p<0.001$ ), and (b) the line represents the regression line for sampling dates when the corn was not at peak growth (DAS 11, 24, and 171), $\log _{10}(y)=\left[3.663 \times \log _{10}(x)\right]+0.822\left(R^{2}=0.80\right.$, $n=24, p<0.001)$.

sidered (DAS 59 and 94), $57 \%$ of the variability in gross $\mathrm{CH}_{4}$ production rates was predicted by the combination of $\mathrm{CO}_{2}$ emissions, net $\mathrm{N}$ mineralization, and net nitrification $(n=14$, $p=0.03$ ).

Gross $\mathrm{CH}_{4}$ oxidation did not differ significantly among sampling dates (Fig. 2b), averaging $1.1 \pm 0.2 \mathrm{mg} \mathrm{C} \mathrm{m}^{-2} \mathrm{~d}^{-1}$ across all measurements $(n=37)$. Rates were marginally significantly higher in rows than in inter-rows $\left(F_{1,3}=6.1\right.$, $p=0.09)$. Gross $\mathrm{CH}_{4}$ oxidation showed a strong positive relationship with gross $\mathrm{CH}_{4}$ production $\left(R^{2}=0.67, n=37\right.$, $p<0.001)$. When gross $\mathrm{CH}_{4}$ production rates exceeded $0.22 \mathrm{mg} \mathrm{C} \mathrm{m}^{-2} \mathrm{~d}^{-1}$, gross $\mathrm{CH}_{4}$ oxidation rates exhibited a tight 1:1 relationship with gross $\mathrm{CH}_{4}$ production rates (slope $=1.06 \pm 0.05 ; R^{2}=0.95, n=27, p<0.001$, Fig. 4b). Below this threshold of gross $\mathrm{CH}_{4}$ production, gross $\mathrm{CH}_{4}$ oxidation was not correlated to gross $\mathrm{CH}_{4}$ production rates alone, but was strongly correlated to the combination of gross $\mathrm{CH}_{4}$ production and soil temperature $\left(R^{2}=0.67, n=10\right.$, $p=0.02$ ); oxidation rates exhibited a negative relationship with soil temperature $\left(R^{2}=0.40, n=10, p=0.05\right)$. Overall, gross oxidation rates were best predicted by the combination of gross $\mathrm{CH}_{4}$ production rates, soil temperature, and $\mathrm{CO}_{2}$ emissions $\left(R^{2}=0.79\right.$, Table 4$)$.

\section{$3.4 \mathrm{CO}_{2}$ emissions}

Carbon dioxide emissions ranged $0.6-10.5 \mathrm{~g} \mathrm{C} \mathrm{m}^{-2} \mathrm{~d}^{-1}$ across the entire data set. Emissions trended higher in the rows than in the inter-rows after the corn germinated, but repeated measures ANOVA showed that $\mathrm{CO}_{2}$ emissions differed significantly among sampling dates $\left(F_{4,56}=80.1\right.$, $p<0.001)$ but not between row and inter-row locations (Fig. 1b). The highest $\mathrm{CO}_{2}$ emissions occurred on DAS 59 and 94 , at the height of the growing season, averag-
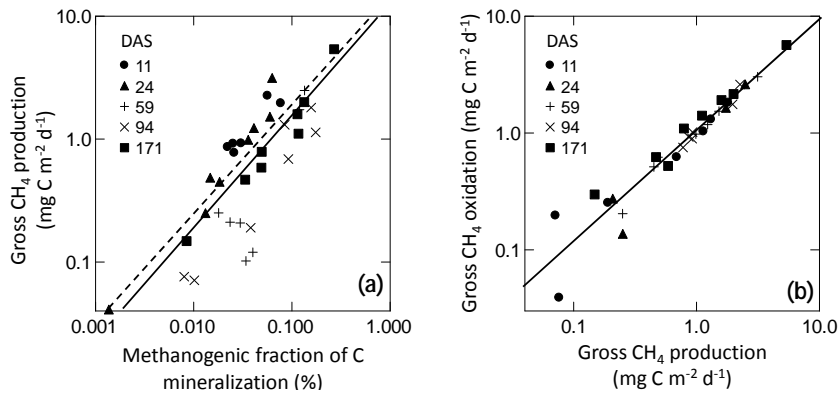

Figure 4. (a) Gross $\mathrm{CH}_{4}$ production rates versus methanogenic fraction of $\mathrm{C}$ mineralization, and (b) gross $\mathrm{CH}_{4}$ oxidation rates versus gross $\mathrm{CH}_{4}$ production rates. Symbols represent sampling on different days after seeding (DAS): circles are DAS 11, triangles are DAS 24, pluses are DAS 59, crosses are DAS 94, and squares are DAS 171. The solid lines represent the regression line for all sampling dates together, (a) $\log _{10}(y)=\left[18.953 \times \log _{10}(x)\right]+2.245\left(R^{2}=0.40, n=37\right.$, $p<0.001) \quad$ and $\quad$ (b) $\quad \log _{10}(y)=\left[1.308 \times \log _{10}(x)\right]-1.028$ $\left(R^{2}=0.67, \quad n=37, \quad p<0.001\right)$. The dashed line represents the regression line for DAS 11, 24, and 171 only, $\log _{10}(y)=\left[22.681 \times \log _{10}(x)\right]+1.904 \quad\left(R^{2}=0.60, \quad n=23\right.$, $p<0.001)$.

ing $6.7 \pm 0.2 \mathrm{~g} \mathrm{C} \mathrm{m}^{-2} \mathrm{~d}^{-1}$; the lowest emissions occurred on DAS 11 and 24 at the beginning of the growing season, averaging $2.6 \pm 0.2 \mathrm{~g} \mathrm{C} \mathrm{m}^{-2} \mathrm{~d}^{-1}$. The variability in $\mathrm{CO}_{2}$ emissions was poorly explained by environmental and soil variables with soil moisture and soil temperature together as the best, yet weak, predictors $\left(R^{2}=0.15\right.$, Table 4$)$.

\section{Discussion}

\section{1 $\quad \mathrm{N}_{2} \mathrm{O}$ dynamics}

Net $\mathrm{N}_{2} \mathrm{O}$ fluxes at our study site were comparable to those reported for other fertilized crop fields (Gelfand et al., 2013; Smith et al., 2011; Stevens and Laughlin, 1998; Nangia et al., 2013; Robertson et al., 2000), averaging $1.5 \pm 0.2 \mathrm{mg} \mathrm{N} \mathrm{m}^{-2} \mathrm{~d}^{-1}$ across the growing season. Prior field estimates of $\mathrm{N}_{2} \mathrm{O}$ yield using ${ }^{15} \mathrm{NH}_{4}$ or ${ }^{15} \mathrm{NO}_{3}$ addition at application rates of $200-300 \mathrm{~kg} \mathrm{~N}^{-1}$ span a wide range from 0.06 to 0.7 (Mosier et al., 1986; Rolston et al., 1976, $1978,1982)$. In contrast, the $\mathrm{N}_{2} \mathrm{O}$ yield varied little throughout the growing season at our site, averaging $0.68 \pm 0.02$, despite significant differences in both net and gross $\mathrm{N}_{2} \mathrm{O}$ fluxes among sampling dates. This is similar to a field estimate of the $\mathrm{N}_{2} \mathrm{O}$ yield for a nearby pasture on the same soil type $\left(0.70 \pm 0.04\right.$; Yang et al., 2011). Soil $\mathrm{NO}_{3}^{-}$concentrations in surface soils $(0-10 \mathrm{~cm}$ depth) were 1-2 orders of magnitude greater in the cornfield than in the pasture, so it is surprising that the $\mathrm{N}_{2} \mathrm{O}$ yields were similar. Soil $\mathrm{NO}_{3}^{-}$concentration was the strongest predictor of $\mathrm{N}_{2} \mathrm{O}$ yield in a US Midwest cornfield soil incubated in the laboratory (Woli et al., 2010). 
Other factors such as soil $\mathrm{pH}$, labile $\mathrm{C}$ availability, or soil aggregation may have played a more important role in controlling the $\mathrm{N}_{2} \mathrm{O}$ yield in our cornfield (Sey et al., 2008).

The best predictors of gross $\mathrm{N}_{2} \mathrm{O}$ production and consumption changed over the growing season, likely reflecting the influence of plant-microbial competition for $\mathrm{N}$ on $\mathrm{N}_{2} \mathrm{O}$ dynamics. This is a novel finding because, to our knowledge, this is the first study that has made repeated measurements of gross $\mathrm{N}_{2} \mathrm{O}$ dynamics over the growing season in the presence of active plant-microbial competition for $\mathrm{N}$. When the corn was actively growing, $89 \%$ of the variability in gross $\mathrm{N}_{2} \mathrm{O}$ production was explained by soil moisture, soil temperature, net $\mathrm{N}$ mineralization, and $\mathrm{CO}_{2}$ emissions together. In contrast, when the corn was not actively growing, both gross $\mathrm{N}_{2} \mathrm{O}$ production and reduction were best predicted by soil $\mathrm{CO}_{2}$ emissions alone. This may reflect the role of $\mathrm{CO}_{2}$ emissions as proxy for the availability of labile $\mathrm{C}$ as an electron donor for denitrification; during the growing season, the contribution of autotrophic respiration to soil $\mathrm{CO}_{2}$ emissions obscured this role. Net $\mathrm{N}$ mineralization was an explanatory variable for gross $\mathrm{N}_{2} \mathrm{O}$ production only during the growing season when plant uptake of $\mathrm{N}$ could have limited $\mathrm{N}_{2} \mathrm{O}$ production.

Overall, gross $\mathrm{N}_{2} \mathrm{O}$ reduction rates were strongly correlated to gross $\mathrm{N}_{2} \mathrm{O}$ production rates. This relationship was also observed in a managed grassland with high soil mineral $\mathrm{N}$ concentrations and net soil $\mathrm{N}_{2} \mathrm{O}$ emissions (Yang et al., 2011), but not in a salt marsh with low mineral $\mathrm{N}$ availability where net $\mathrm{N}_{2} \mathrm{O}$ uptake by soil occurred (Yang and Silver, 2016). The strong relationship between $\mathrm{N}_{2} \mathrm{O}$ production and reduction may have driven the well-constrained $\mathrm{N}_{2} \mathrm{O}$ yields in both this study and the managed grassland study because $\mathrm{N}_{2} \mathrm{O}$ reduction increased proportionally to $\mathrm{N}_{2} \mathrm{O}$ production rates. Additional studies using the trace gas pool dilution technique in the field could elucidate whether or not this relationship holds only in soils with high mineral $\mathrm{N}$ concentrations to drive high rates of $\mathrm{N}_{2} \mathrm{O}$ production.

\section{2 $\mathrm{CH}_{4}$ dynamics}

The small and zero net $\mathrm{CH}_{4}$ fluxes we observed, which are typical of cornfields (Mosier et al., 2006), masked gross $\mathrm{CH}_{4}$ fluxes which were 2 orders of magnitude greater. Net $\mathrm{CH}_{4}$ fluxes were generally undetectable because $\mathrm{CH}_{4}$ oxidation was tightly coupled to methanogenesis, especially at high gross $\mathrm{CH}_{4}$ production rates. The ability of methanotrophs to adjust activity to match, but not exceed, rates of methanogenesis could reflect oxidation of soil-derived $\mathrm{CH}_{4}$ at high concentrations near methanogenic microsites but not atmospheric $\mathrm{CH}_{4}$ at low concentrations in the bulk soil. There are a few mechanisms that could drive a stimulatory effect of high $\mathrm{CH}_{4}$ concentrations on $\mathrm{CH}_{4}$ oxidation without increasing oxidation rates at atmospheric concentrations (Benstead and King, 1997). First, high microsite $\mathrm{CH}_{4}$ concentrations can increase the number of methanotrophs as well as shift the methanotrophic community composition from high affinity type II methanotrophs, who consume $\mathrm{CH}_{4}$ at low concentrations, to low affinity type I methanotrophs, who consume $\mathrm{CH}_{4}$ only at high concentrations, in or near the methanogenic microsites (Bender and Conrad, 1992, 1995). Second, the enzyme affinity of type II methanotrophs can change from high affinity in the presence of atmospheric $\mathrm{CH}_{4}$ concentrations to low affinity at high $\mathrm{CH}_{4}$ concentrations, thereby reducing their capability to oxidize $\mathrm{CH}_{4}$ at low concentrations (Dunfield et al., 1999). Third, high $\mathrm{CH}_{4}$ availability may be needed to stimulate enzyme synthesis (Bender and Conrad, 1992, 1995; Nesbit and Breitenbeck), and thus methanotrophic activity may be induced only near methanogenic microsites and not in the bulk soil. Additional studies investigating gross $\mathrm{CH}_{4}$ dynamics in soil aggregates or through the soil profile could provide insight into the mechanisms coupling $\mathrm{CH}_{4}$ production and consumption. Regardless of the mechanisms, our observations suggest that using in situ methods that preserve spatial variability in soil $\mathrm{CH}_{4}$ concentrations and allow for the occurrence of both $\mathrm{CH}_{4}$ production and oxidation, such as the trace gas pool dilution technique, is important for accurately characterizing $\mathrm{CH}_{4}$ dynamics in soil.

Gross $\mathrm{CH}_{4}$ production rates were strongly positively correlated with the methanogenic fraction of $\mathrm{C}$ mineralization, an index of anaerobic soil microsites where electron acceptors are depleted relative to $\mathrm{C}$ supply (von Fischer and Hedin, 2007). Von Fischer et al. (2007) found that the methanogenic fraction was constrained below $0.04 \%$ and gross $\mathrm{CH}_{4}$ production rates below $1 \mathrm{mg} \mathrm{C} \mathrm{m}^{-2} \mathrm{~d}^{-1}$ in tropical and temperate forest soils with less than $60 \%$ water-filled pore space. Though the slope of the relationship between gross $\mathrm{CH}_{4}$ production rates and the methanogenic fraction observed here was similar to that reported by von Fischer et al. (2007), the maximum methanogenic fraction observed here was nearly 7 times greater. The maximum gross $\mathrm{CH}_{4}$ production rate was also an order of magnitude greater than the maximum rate of $0.5 \mathrm{mg} \mathrm{C} \mathrm{m}^{-2} \mathrm{~d}^{-1}$ reported by von Fischer and Hedin (2002) for a range of unsaturated upland soils in which net $\mathrm{CH}_{4}$ fluxes were near zero ( -0.2 to $0.2 \mathrm{mg} \mathrm{C} \mathrm{m}^{-2} \mathrm{~d}^{-1}$ ). This suggests a higher potential for the development of methanogenic microsites in these drained peatland soils, which are rich in $\mathrm{C}$.

The near-zero net $\mathrm{CH}_{4}$ fluxes measured in our cornfield are consistent with other studies in agricultural systems, but the relatively high gross $\mathrm{CH}_{4}$ oxidation rates we documented challenge the paradigm that agricultural soils have low potential for $\mathrm{CH}_{4}$ oxidation compared to unsaturated soils in natural ecosystems (Bender and Conrad, 1994; Koschorreck and Conrad, 1993; Mosier et al., 1991; Nesbit and Breitenbeck, 1992; Zhuang et al., 2013). Our soils had high $\mathrm{NH}_{4}^{+}$ and $\mathrm{NO}_{3}^{-}$concentrations, which did not limit the ability of methanotrophs to completely consume soil-derived $\mathrm{CH}_{4}$. Undisturbed soils in which $\mathrm{CH}_{4}$ production and consumption occur simultaneously could behave differently than manipu- 
lated soils incubated in the laboratory under conditions to isolate $\mathrm{CH}_{4}$ oxidation from $\mathrm{CH}_{4}$ production, and vice versa. Application of the trace gas pool dilution technique to other agricultural fields could reveal whether or not the tight coupling of $\mathrm{CH}_{4}$ production and consumption rather than low rates of $\mathrm{CH}_{4}$ production and oxidation could be responsible for the general observation of small and near-zero net $\mathrm{CH}_{4}$ fluxes in agricultural ecosystems. A greater understanding of limitations on gross $\mathrm{CH}_{4}$ oxidation under field conditions is needed to accurately predict how land use change will alter soil-atmosphere $\mathrm{CH}_{4}$ exchange and to better manage agricultural soils to be atmospheric $\mathrm{CH}_{4}$ sinks.

Our data provide circumstantial evidence that plants could mediate gross $\mathrm{CH}_{4}$ dynamics in upland soil both directly and indirectly. An increase in plant $\mathrm{C}$ inputs to the soil over the growing season may have directly driven a steady, though not statistically significant, increase in rates of methanogenesis by providing more $\mathrm{C}$ substrate to support methanogenesis. Both gross $\mathrm{CH}_{4}$ production and oxidation rates were approximately 2.5 times greater at DAS 171 compared to DAS 11. This trend in gross $\mathrm{CH}_{4}$ fluxes cannot be explained by changes in environmental variables such as soil temperature, which peaked in the middle of the growing season, and soil moisture, which decreased over the growing season. However, von Fischer and Hedin (2007) showed that methanogenesis was not limited by $\mathrm{C}$ supply in a wide range of upland soils, but rather, it was limited by the number of anaerobic microsites that could support methanogenesis in the soils. Our data also support the latter mechanism controlling methanogenesis: we observed a strong relationship between gross $\mathrm{CH}_{4}$ production and the methanogenic fraction of $\mathrm{C}$ mineralization (an index of the abundance of anaerobic soil microsites) on DAS 11, 24, and 171, when root respiration likely did not contribute significantly to $\mathrm{CO}_{2}$ effluxes. We also observed higher gross $\mathrm{CH}_{4}$ production and oxidation rates in rows than in inter-rows, suggesting that plants could indirectly control methanogenesis through rhizosphere priming, fueling biological $\mathrm{O}_{2}$ demand for $\mathrm{C}$ mineralization (Zhu et al., 2014) that creates a greater number of anaerobic soil microsites supporting methanogenesis.

\section{Conclusions}

Our study demonstrates that the anaerobic processes of $\mathrm{N}_{2} \mathrm{O}$ reduction to $\mathrm{N}_{2}$ and methanogenesis can play important roles in mediating soil-atmosphere greenhouse gas fluxes in upland crop field soils where these processes have previously been discounted. Moreover, despite high soil $\mathrm{NO}_{3}^{-}$and $\mathrm{NH}_{4}^{+}$ concentrations that theoretically inhibit $\mathrm{N}_{2} \mathrm{O}$ reduction to $\mathrm{N}_{2}$ as well as $\mathrm{CH}_{4}$ oxidation, gross $\mathrm{N}_{2} \mathrm{O}$ reduction rates were approximately one-third of gross $\mathrm{N}_{2} \mathrm{O}$ production rates and $\mathrm{CH}_{4}$ oxidation kept pace with methanogenesis that reached relatively high rates for unsaturated soil. Our field measurements of gross $\mathrm{N}_{2} \mathrm{O}$ and $\mathrm{CH}_{4}$ fluxes thus challenge our cur- rent understanding of the controls on the production and consumption of $\mathrm{N}_{2} \mathrm{O}$ and $\mathrm{CH}_{4}$ in upland soils. The strong correlations that gross $\mathrm{N}_{2} \mathrm{O}$ and $\mathrm{CH}_{4}$ fluxes exhibited with soil characteristics and soil $\mathrm{N}$ cycling process rates can help guide controlled studies to investigate the controls on the processes that lead to the production and consumption of $\mathrm{N}_{2} \mathrm{O}$ and $\mathrm{CH}_{4}$. A better understanding of the controls on these processes can help refine modeling efforts to characterize the effects of anoxic microsites in unsaturated soil on greenhouse gas emissions (Riley et al., 2011) and also inform land management decisions to mitigate soil greenhouse gas emissions from crop fields.

Acknowledgements. We appreciate field and lab assistance from Heather Dang, Andrew McDowell, Gavin McNicol, Julia Cosgrove, Rebecca Ryals, Zoe Statman-Weil, Jonathan Treffkorn, Jonathan Lee, Taichi Natake, Ryan Salladay, and Kristina Solheim. Funding was provided by the California Department of Water Resources, by a US National Science Foundation grant (DEB0543558) to W. L. Silver and by the USDA National Institute of Food and Agriculture, McIntire Stennis project (CA-B-ECO-7673MS) to W. L. Silver.

Edited by: A. Ito

\section{References}

Adamsen, A. P. S. and King, G. M.: Methane consumption in temperate and sub-arctic forest soils: rates, vertical zonation, and responses to water and nitrogen, Appl. Environ. Microbiol., 59, 485-490, 1993.

Aronson, E. L. and Helliker, B. R.: Methane flux in non-wetland soils in response to nitrogen addition: a meta-analysis, Ecology, 91, 3242-3251, 2010.

Bedard, C. and Knowles, R.: Physiology, biochemistry, and specific inhibitors of $\mathrm{CH}_{4}, \mathrm{NH}_{4}^{+}$, and co-oxidation by methanotrophs and nitrifiers, Microbiol. Rev., 53, 68-84, 1989.

Bender, M. and Conrad, R.: Kinetics of $\mathrm{CH}_{4}$ oxidation in oxic soils exposed to ambient air or high $\mathrm{CH}_{4}$ mixing ratios, FEMS Microbiol. Ecol., 101, 261-270, 1992.

Bender, M. and Conrad, R.: Methane oxidation activity in various soils and freshwater sediments: occurrence, characteristics, vertical profiles, and distribution on grain-size fractions, J. Geophys. Res.-Atmos., 99, 16531-16540, 1994.

Bender, M. and Conrad, R.: Effect of $\mathrm{CH}_{4}$ concentrations and soil conditions on the induction of $\mathrm{CH}_{4}$ oxidation activity, Soil Biol. Biochem., 27, 1517-1527, 1995.

Benstead, J. and King, G. M.: Response of methanotrophic activity in forest soil to methane availability, FEMS Microbiol. Ecol., 23, 333-340, 1997.

Cai, Y. J., Ding, W. X., and Luo, J. F.: Spatial variation of nitrous oxide emission between interrow soil and interrow plus row soil in a long-term maize cultivated sandy loam soil, Geoderma, 181, 2-10, 2012.

Cheng, W. X., Johnson, D. W., and Fu, S. L.: Rhizosphere effects on decomposition: Controls of plant species, phenology, and fertilization, Soil Sci. Soc. Am. J., 67, 1418-1427, 2003. 
Ciais, P. C., Sabine, C., Bala, G., Bopp, L., Brovkin, V., Canadell, J., Chhabra, A., DeFries, R., Galloway, J., Heimann, M., Jones, C., Le Quèrè, C., Myneni, R. B., Piao, S., and Thornton, P.: Carbon and Other Biogeochemical Cycles, in: Climate Change 2013: The Physical Science Basis. Contribution of Working Group 1 to the Fifth Assessment Report of the Intergovernmental Planel on Climate Change, edited by: Stocker, T. F., Qin, D., Plattner, G.K., Tignor, M., Allen, S. K., Boschung, J., Nauels, A., Xia, Y., Bex, V., and Midgley, P. M., Cambridge University Press, Cambridge, United Kingdom and New York, NY, USA, 465-570, 2013.

Conrad, R.: Soil microorganisms as controllers of atmospheric trace gases $\left(\mathrm{H}_{2}, \mathrm{CO}, \mathrm{CH}_{4}, \mathrm{OCS}, \mathrm{N}_{2} \mathrm{O}\right.$, and $\left.\mathrm{NO}\right)$, Microbiol. Rev., 60, 609-640, 1996.

Drexler, J. Z., de Fontaine, C. S., and Deverel, S. J.: The legacy of wetland drainage on the remaining peat in the Sacramento-San Joaquin Delta, California, USA, Wetlands, 29, 372-386, 2009.

Dunfield, P., Knowles, R., Dumont, R., and Moore, T. R.: Methane production and consumption in temperate and sub-arctic peat soils: response to temperature and $\mathrm{pH}$, Soil Biol. Biochem., 25, 321-326, 1993.

Dunfield, P. F., Liesack, W., Henckel, T., Knowles, R., and Conrad, R.: High-affinity methane oxidation by a soil enrichment culture containing a type II methanotroph, Appl. Environ. Microbiol., 65, 1009-1014, 1999.

Dutaur, L. and Verchot, L. V.: A global inventory of the soil $\mathrm{CH}_{4}$ sink, Global Biogeochem. Cy., 21, GB4013, doi: 10.1029/2006GB002734, 2007.

Firestone, M. K., Firestone, R. B., and Tiedje, J. M.: Nitrous oxide from soil denitrification: factors controlling its biological production, Science, 208, 749-751, 1980.

Fisher, R., Lowry, D., Wilkin, O., Sriskantharajah, S., and Nisbet, E. G.: High-precision, automated stable isotope analysis of atmospheric methane and carbon dioxide using continuous-flow isotope-ratio mass spectrometry, Rapid Commun. Mass Sp., 20, 200-208, 2006.

Gelfand, I., Sahajpal, R., Zhang, X. S., Izaurralde, R. C., Gross, K. L., and Robertson, G. P.: Sustainable bioenergy production from marginal lands in the US Midwest, Nature, 493, 514-517, 2013.

Groffman, P. M., Altabet, M. A., Bohlke, J. K., Butterbach-Bahl, K., David, M. B., Firestone, M. K., Giblin, A. E., Kana, T. M., Nielsen, L. P., and Voytek, M. A.: Methods for measuring denitrification: Diverse approaches to a difficult problem, Ecol. Appl., 16, 2091-2122, 2006.

Gulledge, J. and Schimel, J. P.: Low concentration kinetics of atmospheric $\mathrm{CH}_{4}$ oxidation in soil and mechanism of $\mathrm{NH}_{4}^{+}$inhibition, Appl. Environ. Microbiol., 64, 4291-4298, 1998.

Gulledge, J., Doyle, A. P., and Schimel, J. P.: Different $\mathrm{NH}_{4}^{+}$inhibition patterns of soil $\mathrm{CH}_{4}$ consumption: A result of distinct $\mathrm{CH}_{4}$ oxidizer populations across sites?, Soil Biol. Biochem., 29, 13-21, 1997.

Haile-Mariam, S., Collins, H. R., and Higgins, S. S.: Greenhouse gas fluxes from an irrigated sweet corn (Zea mays L.)-potato (Solanum tuberosum L.) rotation, J. Environ. Qual., 37, 759-771, 2008.

Hatala, J. A., Detto, M., Sonnentag, O., Deverel, S. J., Verfaille, J., and Baldocchi, D. D.: Greenhouse gases $\left(\mathrm{CO}_{2}, \mathrm{CH}_{4}, \mathrm{H}_{2} \mathrm{O}\right)$ fluxes from drained and flooded agricultural peatlands in the
Sacramento-San Joaquin Delta. Agric. Ecosyst. Environ., 150, 1-18, 2012.

Ingebritsen, S. E. and Ikehara, M. E.: Sacramento-San Joaquin Delta: The sinking heart of the state, in: Land Subsidence in the United States, edited by: Galloway, D., Jones, D. R., and Ingrebritsen, S. E., US Geological Survey, Reston, Virginia, 83-94, 1999.

Jackel, U., Schnell, S., and Conrad, R.: Effect of moisture, texture and aggregate size of paddy soil on production and consumption of $\mathrm{CH}_{4}$, Soil Biol. Biochem., 33, 965-971, 2001.

Jang, I., Lee, S., Zoh, K. D., and Kang, H.: Methane concentrations and methanotrophic community structure influence the response of soil methane oxidation to nitrogen content in a temperate forest, Soil Biol. Biochem., 43, 620-627, 2011.

Kessavalou, A., Mosier, A. R., Doran, J. W., Drijber, R. A., Lyon, D. J., and Heinemeyer, O.: Fluxes of carbon dioxide, nitrous oxide, and methane in grass sod and winter wheat-fallow tillage management, J. Environ. Qual., 27, 1094-1104, 1998.

King, G. M. and Schnell, S.: Effect of increasing atmospheric methane concentration on ammonium inhibition of soil methane consumption, Nature, 370, 282-284, 1994.

Koschorreck, M. and Conrad, R.: Oxidation of atmospheric methane in soil: measurements in the field, in soil cores and in soil samples, Global Biogeochem. Cy., 7, 109-121, 1993.

Matthias, A. D., Yarger, D. N., and Weinbeck, R. S.: Numerical evaluation of chamber methods for determining gas fluxes, Geophys. Res. Lett., 5, 765-768, 1978.

Mosier, A., Schimel, D., Valentine, D., Bronson, K., and Parton, W.: Methane and nitrous oxide fluxes in native, fertilized and cultivated grasslands, Nature, 350, 330-332, 1991.

Mosier, A. R., Guenzi, W. D., and Schweizer, E. E.: Soil losses of dinitrogen and nitrous oxide from irrigated crops in northeastern Colorado, Soil Sci. Soc. Am. J., 50, 344-348, 1986.

Mosier, A. R., Halvorson, A. D., Reule, C. A., and Liu, X. J. J.: Net global warming potential and greenhouse gas intensity in irrigated cropping systems in northeastern Colorado, J. Environ. Qual., 35, 1584-1598, 2006.

Myhre, G., Shindell, D., Breon, F.-M., Collins, W., Fuglestvedt, J., Huang, J., Koch, D., Lamarque, J.-F., Lee, D., Mendoza, B., Nakajima, T., Robock, A., Stephens, G., Takemura, T., and Zhang, H.: Anthropogenic and Natural Radiative Forcing, in: Climate Change 2013: The Physical Science Basis. Contribution of Working Group 1 to the Fifth Assessment Report of the Intergovernmental Planel on Climate Change, edited by: Stocker, T. F., Qin, D., Plattner, G.-K., Tignor, M., Allen, S. K., Boschung, J., Nauels, A., Xia, Y., Bex, V., and Midgley, P. M., Cambridge University Press, Cambridge, United Kingdom and New York, NY, USA, 659-740, 2013.

Nangia, V., Sunohara, M. D., Topp, E., Gregorich, E. G., Drury, C. F., Gottschall, N., and Lapen, D. R.: Measuring and modeling the effects of drainage water management on soil greenhouse gas fluxes from corn and soybean fields, J. Environ. Manage., 129, 652-664, 2013.

Nesbit, S. P. and Breitenbeck, G. A.: A laboratory study of factors influencing methane uptake by soils, Agric. Ecosyst. Environ., 41, 39-54, 1992.

Reay, D. S. and Nedwell, D. B.: Methane oxidation in temperate soils: effects of inorganic N, Soil Biol. Biochem., 36, 2059-2065, 2004. 
Riley, W. J., Subin, Z.M., Lawrence, D. M., Swenson, S. C., Torn, M. S., Meng, L., Mahowald, N. M., and Hess, P.: Barriers to predicting changes in global terrestrial methane fluxes: analyses using CLM4Me, a methane biogeochemistry model integrated in CESM, Biogeosciences, 8, 1925-1953, doi:10.5194/bg-8-19252011, 2011.

Robertson, G. P., Paul, E. A., and Harwood, R. R.: Greenhouse gases in intensive agriculture: Contributions of individual gases to the radiative forcing of the atmosphere, Science, 289, 1922$1925,2000$.

Rolston, D. E., Fried, M., and Goldhamer, D. A.: Denitrification measured directly from nitrogen and nitrous oxide gas fluxes, Soil Sci. Soc. Am. J., 40, 259-266, 1976.

Rolston, D. E., Hoffman, D. L., and Toy, D. W.: Field measurement of denitrification. 1. Flux of $\mathrm{N}_{2}$ and $\mathrm{N}_{2} \mathrm{O}$, Soil Sci. Soc. Am. J., 42, 863-869, 1978.

Rolston, D. E., Sharpley, A. N., Toy, D. W., and Broadbent, F. E.: Field measurement of denitrification, 3. Rates during irrigation cycles, Soil Sci. Soc. Am. J., 46, 289-296, 1982.

Schlesinger, W. H.: On the fate of anthropogenic nitrogen, Proc. Natl. Acad. Sci. USA, 106, 203-208, 2009.

Sexstone, A. J., Revsbech, N. P., Parkin, T. B., and Tiedje, J. M.: Direct measurement of oxygen profiles and denitrification rates in soil aggregates, Soil Sci. Soc. Am. J., 49, 645-651, 1985.

Sey, B. K., Manceur, A. M., Whalen, J. K., Gregorich, E. G., and Rochette, P.: Small-scale heterogeneity in carbon dioxide, nitrous oxide and methane production from aggregates of a cultivated sandy-loam soil, Soil Biol. Biochem., 40, 2468-2473, 2008.

Smith, D. R., Hernandez-Ramirez, G., Armstrong, S. D., Bucholtz, D. L., and Stott, D. E.: Fertilizer and tillage management impacts on non-carbon dioxide greenhouse gas emissions, Soil Sci. Soc. Am. J., 75, 1070-1082, 2011.

Stevens, R. J. and Laughlin, R. J.: Measurement of nitrous oxide and di-nitrogen emissions from agricultural soils, Nutr. Cycl. Agroecosyst., 52, 131-139, 1998.

Stevens, R. J., Laughlin, R. J., and Malone, J. P.: Soil pH affects the processes reducing nitrate to nitrous oxide and di-nitrogen, Soil Biol. Biochem., 30, 1119-1126, 1998.

Teh, Y. A., Silver, W. L., and Conrad, M. E.: Oxygen effects on methane production and oxidation in humid tropical forest soils, Glob. Change Biol., 11, 1283-1297, 2005. von Fischer, J. C. and Hedin, L. O.: Separating methane production and consumption with a field-based isotope pool dilution technique, Global Biogeochem. Сy., 16, 1034, doi:10.1029/2001gb001448, 2002.

von Fischer, J. C. and Hedin, L. O.: Controls on soil methane fluxes: Tests of biophysical mechanisms using stable isotope tracers, Global Biogeochem. Cy., 21, GB2007, doi:10.1029/2006gb002687, 2007.

Wang, Z. P. and Ineson, P.: Methane oxidation in a temperate coniferous forest soil: effects of inorganic N, Soil Biol. Biochem., 35 , 427-433, 2003.

Weier, K. L., Doran, J. W., Power, J. F., and Walters, D. T.: Denitrification and the dinitrogen nitrous oxide ratio as affected by soil water, available carbon, and nitrate, Soil Sci. Soc. Am. J., 57, 66-72, 1993.

Well, R. and Butterbach-Bahl, K.: Comments on "A test of a field-based ${ }^{15} \mathrm{~N}$-nitrous oxide pool dilution technique to measure gross $\mathrm{N}_{2} \mathrm{O}$ production in soil" by Yang et al. (2011), Global Change Biology, 17, 3577-3588, Glob. Change Biol., 19, 133135, 2013.

Woli, K. P., David, M. B., Darmody, R. G., Mitchell, C. A., and Smith, C. M.: Assessing the nitrous oxide mole fraction of soils from perennial biofuel and corn-soybean fields, Agric. Ecosyst. Environ., 138, 299-305, 2010.

Yang, W. H. and Silver, W. L.: Gross nitrous oxide production drives net nitrous oxide fluxes across a salt marsh landscape, Glob. Change Biol., in press, doi:10.1111/gcb.13203, 2016.

Yang, W. H., Teh, Y. A., and Silver, W. L.: A test of a field-based ${ }^{15} \mathrm{~N}$-nitrous oxide pool dilution technique to measure gross $\mathrm{N}_{2} \mathrm{O}$ production in soil, Glob. Change Biol., 17, 3577-3588, 2011.

Yang, W. H., Teh, Y. A., and Silver, W. L.: Measuring gross $\mathrm{N}_{2} \mathrm{O}$ production in soil: a reply to Well and Butterbach-Bahl, Glob. Change Biol., 19, 985-987, 2013.

Zhu, B., Gutknecht, J. L. M., Herman, D. J., Keck, D. C., Firestone, M. K., and Cheng, W. X.: Rhizosphere priming effects on soil carbon and nitrogen mineralization, Soil Biol. Biochem., 76, 183-192, 2014.

Zhuang, Q. L., Chen, M., Xu, K., Tang, J. Y., Saikawa, E., Lu, Y. Y., Melillo, J.M., Prinn, R. G., and McGuire, A. D.: Response of global soil consumption of atmospheric methane to changes in atmospheric climate and nitrogen deposition, Global Biogeochem. Cy., 27, 650-663, 2013. 